\title{
Robust Passivity Preserving Parametric Model Order Reduction using Matrix Interpolation
}

\author{
Elizabeth Rita Samuel*, Francesco Ferranti*, Luc Knockaert*, Tom Dhaene** \\ * Ghent University - IBBT, Gaston Crommenlaan 8 Bus 201, B-9050 Gent, \\ Belgium,email: \{elizabeth.ritasamuel, francesco.ferranti, luc.knockaert, \\ tom.dhaene\}@ugent.be
}

\begin{abstract}
We present a novel robust parametric model order reduction method using matrix interpolation and common projection matrix. The design space is sampled over an estimation grid and transformation matrices are computed at the estimation points using a projection-based passivity preserving truncated balanced realization. A common projection matrix is obtained by the truncation of the singular values of the merged transformation matrices obtained from the estimation points in the design space. Finally, the reduced system matrices are interpolated using positive interpolation schemes to obtain a guaranteed passive parametric reduced model. The proposed technique is validated using pertinent numerical results.
\end{abstract}

Keywords: Robust, Parametric model order reduction, Singular values, Passivity, Interpolation.

\section{INTRODUCTION}

Large scale systems are present in many fields of engineering and for many applications, such as circuit simulation and timedependent partial differential equation (PDE) control problems, where the internal dimension of the system is quite large, with respect to the number of input and output ports. In these largescale settings, the system dimension makes computations intractable due to memory, time limitations and ill-conditioning. The common approach to overcome this is by means of model order reduction that has similar response characteristics as the original system with a much lower storage requirement and evaluation time. The resulting reduced model can eventually replace the original system as a component in a large simulation or it can be used to develop a low dimensional controller suitable for real time applications.

Therefore, model order reduction (MOR) techniques as in Gallivan et al. [1994], [Feldmann and R. Freund, 1995], Gallivan et al. [1996], Odabasioglu et al. [1998], Knockaert and De Zutter [2000], are crucial when dealing with robust design issues in order to reduce the complexity and computational cost of the simulations, while retaining the important physical features of the original large scale system.

Over the years, active research has been focused on model order reduction. Two main classes of MOR methods can be distinguished: 1) moment matching methods, and 2) balanced and Hankel norm methods. The moment matching methods for large-scale problems have led to the use of Krylov and rational Krylov subspace projection methods. The importance of producing passive or positive-real reduced models resulted in several algorithms that preserve passivity of $R L C$ circuits e.g. Odabasioglu et al. [1998] and Knockaert and De Zutter [2000]. As Krylov techniques fail to generate models with provable error bounds, Kamon et al. [2000], the balanced and Hankel norm approaches have gained attention in the MOR research area. Balanced and Hankel norm reduction methods, already well-developed in the control literature, Glover [1984], have a

\footnotetext{
* This work was supported by the Research Foundation Flanders (FWO).
}

very close connection to the singular value decomposition and have been receiving renewed attention in the electronic design automation community. These methods preserve asymptotic stability and allow for global error bounds. As they rely upon dense matrix computations they do not scale well in terms of computational efficiency and numerical stability. A strong current trend aims at combining these two classes of methods and their corresponding advantages.

MOR techniques perform model order reduction only with respect to the frequency or Laplace variable. It is also essential to analyze the response of a circuit as a function of design parameters, such as geometrical features and other characteristics during the circuit design synthesis of large-scale applications. Parametric model order reduction (PMOR) methods are well suited for such design activities as they can reduce large systems of equations with respect to both frequency and other design parameters.

Different types of PMOR methods have developed over the last years. Multiparameter moment-matching methods presented in Gunupudi et al. [2003], Daniel et al. [2004] use a subspace projection approach, but they suffer from oversize when the number of moments to match is high, either because high accuracy is required or because the number of parameters is large. Passivity preservation for non-affine parameters is presented in Farle et al. [2011] proposes a novel model order reduction technique which preserves passivity but the algorithm requires Cholesky factorization.The technique presented in Ferranti et al. [2010] combines traditional passivity-preserving MOR methods and positive interpolation schemes. A PMOR method based on a parameterization process of matrices generated by electromagnetic (EM) methods and projection subspaces is proposed in Ferranti et al. [2011]. In Ferranti et al. [2010, 2011] the overall passivity of parametric reduced order models (ROMs) is guaranteed over the design space of interest. In Panzer et al. [2010], Eid et al. [2011] a matrix interpolation-based PMOR is presented. A set of reduced system matrices in a common subspace is computed and interpolated to generate a parametric reduced order model. This technique avoids the oversize prob- 
lem of multiparameter moment matching based algorithms, but the reduced system matrices needed for interpolation must have the same reduced order and must be post processed for the reprojection onto the common subspace, which is needed for matrix interpolation. The passivity of parametric reduced order model is not guaranteed.

This paper proposes a novel PMOR technique that enhances and improves the method of Panzer et al. [2010], Eid et al. [2011] by using common projection matrices over the entire design space and passivity preserving parameterization schemes. The modified Smith technique, Gugercin et al. [2001], Wong et al. [2006], is used to compute efficiently the grammians of the large system and then similarity transformation matrices are generated using the projection-based passive truncated balanced realization (TBR), Yan et al. [2007]. The estimation grid and validation grid are chosen for the design space and transformation matrices are computed over the estimation points in the design space. The truncation of the singular values of the merged transformation matrices generates a common projection matrix for the entire design space. A passive parametric reduced model is then obtained by interpolation of the reduced system matrices using positive interpolation schemes.

The paper is organized as follows. Section 2 gives an overview of the Smith technique and the projection-based TBR. Section 3 describes the generation of common projection matrices. Next, multivariate interpolation schemes and passivity-preservation are described in Section 4. Finally some pertinent examples validate the proposed technique in Section 5.

\section{PROJECTION-BASED BALANCED TRUNCATION}

In control theory, eigenvalues or poles define the system stability, whereas Hankel singular values define the energy of each state of the system. Keeping the dominant energy states of a system, preserves most of its characteristics in terms of stability, frequency and time responses. This is the rationale for the use of projection-based TBR in model order reduction. The major advantage of TBR is that it can give a deterministic global bound for the approximation error and can produce nearly optimal models in terms of error and model sizes.

For the projection-based balanced truncation, let us consider a parametric dynamical system with design parameters $\mathbf{g}=$ $\left(g^{(1)}, \ldots, g^{(N)}\right)$ in the descriptor state-space form :

$$
\begin{aligned}
\mathbf{C}(\mathbf{g}) \frac{d \mathbf{x}(t, \mathbf{g})}{d t} & =-\mathbf{G}(\mathbf{g}) \mathbf{x}(t, \mathbf{g})+\mathbf{B u}(t) \\
\mathbf{y}(t, \mathbf{g}) & =\mathbf{L}^{\prime} \mathbf{x}(t, \mathbf{g})+\mathbf{D u}(t)
\end{aligned}
$$

where' is the transpose of the matrix. The fast and efficient modified Smith technique, Gugercin et al. [2001], Wong et al. [2006] is used in this paper to find the controllability grammian $\left(W_{c}\right)$ and the observability grammian $\left(W_{o}\right)$ of a large scale system. For the state-space model (1), the generalized grammians are defined as the unique solutions of the linear equations

$$
\begin{aligned}
& -\mathbf{C}(\mathbf{g}) \mathbf{W}_{\mathbf{c}} \mathbf{G}(\mathbf{g})^{\prime}-\mathbf{G}(\mathbf{g}) \mathbf{W}_{\mathbf{c}} \mathbf{C}(\mathbf{g})^{\prime}+\mathbf{B} \mathbf{B}^{\prime}=0 \\
& -\mathbf{C}(\mathbf{g})^{\prime} \mathbf{W}_{\mathbf{o}} \mathbf{G}(\mathbf{g})-\mathbf{G}(\mathbf{g})^{\prime} \mathbf{W}_{\mathbf{o}} \mathbf{C}(\mathbf{g})+\mathbf{L} \mathbf{L}^{\prime}=0
\end{aligned}
$$

For every real scalar $p<0$, equation (2) can be written as:

$$
\mathbf{A}_{p} \mathbf{W}_{\mathbf{c}} \mathbf{A}_{p}^{\prime}-\mathbf{W}_{\mathbf{c}}+\mathbf{B}_{p} \mathbf{B}_{p}^{\prime}=0
$$

where $\mathbf{A}_{p}=(p \mathbf{C}(g)-\mathbf{G}(g))^{-1}(p \mathbf{C}(g)+\mathbf{G}(g))$, and $\mathbf{B}_{p}=$ $\sqrt{(-2 p)}(p \mathbf{C}(g)-\mathbf{G}(g))^{-1} \mathbf{B}$. Hence the controllability grammian can be computed as

$$
\mathbf{W}_{\mathbf{c}} \approx \sum_{j=0}^{k-1} \mathbf{A}_{p}^{j} \mathbf{B}_{p} \mathbf{B}_{p}^{\prime}\left(\mathbf{A}_{p}^{\prime}\right)^{j}
$$

Similarly, taking $\tilde{\mathbf{A}}_{p}=(p \mathbf{C}(g)+\mathbf{G}(g))(p \mathbf{C}(g)-\mathbf{G}(g))^{-1}$, and $\mathbf{L}_{p}=\sqrt{(-2 p)} \mathbf{L}(p \mathbf{C}(g)-\mathbf{G}(g))^{-1}$, the observability grammian $\mathbf{W}_{\mathbf{o}}$ can be computed. The value of $k$ in (5) can be found from the convergence criterion:

$$
\frac{\left\|\mathbf{W}_{c}^{k-1}-\mathbf{W}_{c}^{k}\right\|_{2}}{\left\|\mathbf{W}_{c}^{k-1}\right\|_{2}} \leq \text { threshold }
$$

The square root of the eigenvalues of the product of the grammians produces the Hankel singular values as shown:

$$
\sqrt{\operatorname{eig}\left(\mathbf{W}_{\mathbf{c}} \mathbf{W}_{\mathbf{o}}\right)}=\Sigma
$$

where $\Sigma=\left[\sigma_{1}, \sigma_{2}, \ldots ., \sigma_{n}\right]$ with $\sigma_{1} \geq \sigma_{2} \ldots \ldots . \geq \sigma_{n}$ where $\sigma_{1}, \sigma_{2}, \ldots ., \sigma_{n}$ are called the Hankel singular values of the system, which quantify its reachability and observability. $q$ can be defined for a desired maximal error.

$$
\|\mathbf{Y}-\hat{\mathbf{Y}}\|_{\infty} \leq 2 \sum_{i=q+1}^{n} \sigma_{i}
$$

where $\mathbf{Y}$ and $\hat{\mathbf{Y}}$ represent the frequency response of the original model and the reduced model.

Once the grammians and the reduced order of the system are computed, the similarity transformation matrix $T$ is computed from the grammians by Cholesky decomposition and SVD as follows:

$$
\begin{aligned}
\mathbf{W}_{\mathbf{c}} & =\mathbf{L}_{c} \mathbf{L}_{c}^{\prime} \\
\mathbf{W}_{\mathbf{o}} & =\mathbf{L}_{o} \mathbf{L}_{o}^{\prime} \\
\mathbf{U} \Sigma \mathbf{V}^{\prime} & =\operatorname{svd}\left(\mathbf{L}_{c}^{\prime} \mathbf{C}^{\prime} \mathbf{L}_{o}\right)
\end{aligned}
$$

Based on $q$ the reduced order as defined in (8), the $\Sigma$ and $\mathbf{U}$ can be truncated to $\Sigma_{q}$ and $\mathbf{U}_{q}$ respectively for computing the transformation matrix $\mathbf{T}_{q}$ as follows:

$$
\begin{aligned}
\mathbf{U} & =\left[\mathbf{U}_{n \times q} \mathbf{U}_{n \times n-q}\right] \\
\Sigma & =\left[\begin{array}{cc}
\Sigma_{q} & 0 \\
0 & \Sigma_{n-q}
\end{array}\right] \\
\mathbf{T}_{q} & =\mathbf{L}_{c} \mathbf{U}_{q} \Sigma_{q}^{-1 / 2}
\end{aligned}
$$

Thus, $\mathbf{T}_{q}$ spans the dominant subspace.

Although TBR-like methods are accurate globally, they cannot generate a reduced model with exact local behavior at low frequencies, unlike what happens in moment matching approaches, where an expansion point can be chosen at frequency 0, Yan et al. [2007]. To overcome this, a zero-order moment is added to the projection matrix of the projection-based TBR method as follows:

$$
\begin{gathered}
\mathbf{G M}_{\mathbf{0}}=\mathbf{B} \\
\mathbf{T}=\left[\mathbf{M}_{\mathbf{0}}, \mathbf{T}_{q}\right]
\end{gathered}
$$

Here $\mathbf{M}_{\mathbf{0}}$ is the zero-order moment. Now, the reduced model has both global accuracy and exact low frequency behavior as the transformation matrix $\mathbf{T}$ is a union of $\mathbf{M}_{\mathbf{0}}$ and $\mathbf{T}_{q}$. Next the transformation matrix is orthonormalized to obtain the projection matrix in order to generate the reduced model by congruence transformation. In this paper we orthonormalize 
a common transformation matrix for the entire design space to get a common projection matrix. This is explained in the following section.

\section{COMMON PROJECTION MATRIX COMPUTATION}

In order to find a common projection matrix first the design space has to be sampled as described in Ferranti et al. [2011], containing all parameters except frequency. Two data grids are used in the process of modeling: an estimation grid and a validation grid as shown in Fig. 1. Parametric reduced models

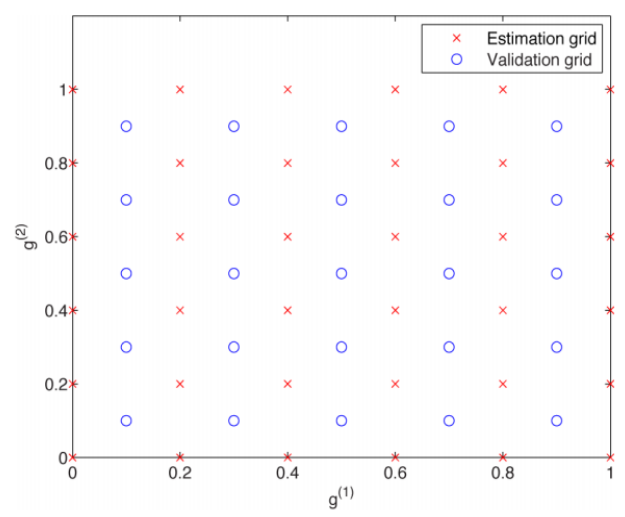

Fig. 1. Example of sampled estimation and validation design space grids.

are estimated globally using the estimation grid and are validated over the validation grid. As described in Section 2, a set of similarity transformation matrices are computed at the estimation points. The similarity transformation matrices will have different dimensions depending on the truncation of the Hankel singular values based on the error-bound. To compute the common projection matrix, the transformation matrices are computed over the estimated grid and are merged by column stacking.

$$
\mathbf{T}_{\text {union }}=\left[T_{1}, T_{2}, \ldots . . T_{\text {Est }_{p t}}\right]
$$

where $E s t_{p t}$ is the number of estimation points considered in the design space. The dimension of $\mathbf{T}_{\text {union }}$ is $n \times w$ where $n$ is the order of the original system and $w=\left(q_{1}+q_{2} \ldots+q_{E s t}\right)$ with $q_{i}$ the reduced order determined as defined in (8) for the $i-t h$ estimation point in the design space. Then, the (economy sized)singular value decomposition (svd) is computed for the union of the projection matrices

$$
\mathbf{U} \Sigma \mathbf{V}^{\prime}=\operatorname{svd}\left(\mathbf{T}_{\text {union }}\right)
$$

It is seen that $\mathbf{U}, \mathbf{V}, \Sigma$ are respectively $n \times w, w \times w$ and $w \times w$ matrices. A common reduced order $r$ for a cell is defined based on the first $r$ significant singular values, by setting a threshold to the ratio of the singular values with respect to the largest singular value.

$$
\begin{aligned}
\sigma_{\max } & =\sigma_{1} \\
\frac{\sigma_{i}}{\sigma_{\max }} & \geq \text { threshold }_{\sigma}, i=1,2, \ldots \ldots ., r
\end{aligned}
$$

Thus a common projection matrix $\mathbf{Q}_{\text {comm }}$ is obtained by the QR orthonormalization of the common transformation matrix, $\mathbf{T}_{\text {comm }}$.

$$
\begin{gathered}
\mathbf{T}_{\text {comm }}=\mathbf{U}_{r} \Sigma_{r} \mathbf{V}_{r}{ }^{\prime} \\
\mathbf{Q}_{\text {comm }}=\mathbf{U}_{r}
\end{gathered}
$$

where $\mathbf{U}_{r}, \Sigma_{r}$ and $\mathbf{V}_{r}$ have the truncated dimension $n \times r$, $r \times r$ and $r \times r$ respectively. The congruence transformation using $\mathbf{Q}_{\text {comm }}$ on the original models over the estimation grid of the design space gives a set of reduced system matrices. Using global approach, this $\mathbf{Q}_{\text {comm }}$ is used over the entire design space. In Leung and Khazaka [2005] svd is used to construct the dominant subspace, but the circuit equation has to be discretized initially to obtain the PMOR. Also a similar approach is used in Li et al. [2005] where svd is applied on the sensitivity matrices of the system.

It is assumed that a fixed discretization mesh is used for the system under study and it is independent of the specific design parameter values, Ferranti et al. [2011]. The size of the system matrices as well as the numbering of the mesh nodes and mesh edges are preserved. The mesh is only locally stretched or shrunk when shape parameters are modified. In general, the global coordinates of the nodes as well as the length and orientation of the edges of the topologically fixed mesh change when shape parameters change, however, these changes neither introduce new state variables nor eliminate existing state variables. The matrices $\mathbf{B}, \mathbf{L}^{\prime}$ are uniquely determined by the circuit topology and therefore remain constant, while the matrices $\mathbf{C}$ and $\mathbf{G}$ are defined as functions of the design parameters. Starting from a set of models in the estimation design space grid that are generated with respect to a common space and using common projection matrices, it can be shown that all the reduced system matrices in the estimation grid are in the same subspace and can be interpolated.

\section{MULTIVARIATE INTERPOLATION}

After computing the reduced matrices, they are interpolated to build a parametric ROM. Tensor product, Cheney [1986] or tessellation methods, Watson [1981] can be used to realize multivariate interpolation. Any interpolation scheme based on a class of positive interpolation operators, Ferranti et al. [2010] can be used, e.g., multilinear, and simplicial methods, Weiser and Zarantonello [1988], to preserve overall passivity as described in what follows.

For example considering multilinear interpolation, each interpolated matrix $\mathbf{J}\left(g^{(1)}, \ldots, g^{(N)}\right)$, can be written as

$$
\begin{gathered}
\mathbf{J}\left(g^{(1)}, \ldots, g^{(N)}\right)=\sum_{k_{1}=1}^{K_{1}} \cdots \sum_{k_{N}=1}^{K_{N}} \mathbf{J}_{\left(g_{k_{1}}^{(1)}, \ldots, g_{k_{N}}^{(N)}\right)} \\
l_{k_{1}}\left(g^{(1)}\right) \cdots l_{k_{N}}\left(g^{(N)}\right)
\end{gathered}
$$

where the interpolation kernel $l_{k_{i}}\left(g^{(i)}\right)$ is a scalar function satisfying the following constraints

$$
\begin{gathered}
0 \leq l_{k_{i}}\left(g^{(i)}\right) \leq 1 \\
l_{k_{i}}\left(g^{(i)}\right)=\delta_{k_{i}} \\
\sum_{k_{i}}^{K_{i}} l_{k_{i}}\left(g^{(i)}\right)=1
\end{gathered}
$$

It can be noted that the interpolation kernel functions of these interpolation methods only depend on the design space grid points and their computation does not require the prior solution of a linear system to impose an interpolation constraint. 
These positive interpolation schemes have already been used in Ferranti et al. [2010], where a parametric macromodel is built by interpolating a set of ROMs treated as input-output systems, while preserving overall stability and passivity. Therefore, interpolating systems, matrices or scalars does not make any difference for these interpolation kernel functions.

When performing transient analysis, stability and passivity must be guaranteed. It is known that, while a passive or positive-real system is also stable, the reverse is not necessarily true, Rohrer and Nosrati [1981], which is crucial when the macromodel is to be utilized in a time domain simulator. Passive systems cannot generate more energy than they absorb through their ports. When the system is terminated on any arbitrary passive load, none of them will cause the system to become unstable, Weinberg [1962], A. Guillemin [1957].

For projection-based TBR, the original systems are assumed to be in the descriptor state space form (1) satisfying the following conditions:

$$
\begin{gathered}
\mathbf{G}+s \mathbf{C} \text { is a regular matrix pencil } \\
\mathbf{C}=\mathbf{C}^{\prime} \geq 0 \\
\mathbf{G}+\mathbf{G}^{\prime} \geq 0 \\
\mathbf{B}=\mathbf{L}
\end{gathered}
$$

Then the passivity of the system with transfer function $\mathbf{Y}(s)=$ $\mathbf{L}^{\prime}(s \mathbf{C}+\mathbf{G})^{-1} \mathbf{B}$ is guaranteed, Freund [2000]. For this specific format, projection-based TBR methods guarantee the passivity of the reduced model built by congruence transformation.

$$
\begin{gathered}
\mathbf{C}_{r}(\mathbf{g})=\mathbf{Q}_{\text {comm }}{ }^{\prime} \mathbf{C}(\mathbf{g}) \mathbf{Q}_{\text {comm }} \geq 0 \\
\mathbf{G}_{r}(\mathbf{g})=\mathbf{Q}_{\text {comm }}{ }^{\prime} \mathbf{G}(\mathbf{g}) \mathbf{Q}_{\text {comm }} \geq 0 \\
\mathbf{B}_{r}(\mathbf{g})=\mathbf{Q}_{\text {comm }}{ }^{\prime} \mathbf{B}(\mathbf{g}) \\
\mathbf{L}_{r}(\mathbf{g})=\mathbf{Q}_{\text {comm }}{ }^{\prime} \mathbf{L}(\mathbf{g})
\end{gathered}
$$

As congruence transformations preserve the definiteness of matrices, the reduced $\mathbf{G}_{r}, \mathbf{C}_{r}$ are also positive semidefinite. Since any nonnegative linear combination of positive (semidefinite) definite real matrices is a positive (semidefinite) definite real matrix, stability and passivity are preserved over the entire design space if positive interpolation operators are used to interpolate reduced matrices.

A flowchart that describes the different steps of the proposed technique is shown in Fig.2.

\section{NUMERICAL RESULTS}

Some numerical examples are used to demonstrate the accuracy and efficiency of the proposed PMOR technique. Let us define the weighted RMS error which is used to validate the accuracy of the results:

$$
\begin{array}{r}
\operatorname{Err}\left(\mathbf{Y}_{1}(s), \mathbf{Y}_{2}(s)\right)=\sqrt{\frac{\sum_{k=1}^{K_{s}} \sum_{i=1}^{P_{\text {in }}} \sum_{j=1}^{P_{\text {out }}} \frac{\left|Y_{1,(i j)}\left(s_{k}\right)-Y_{2,(i j)}\left(s_{k}\right)\right|^{2}}{W_{(i j)}\left(s_{k}\right)}}{P_{\text {in }} P_{\text {out }} K_{s}}} \\
W_{(i j)}\left(s_{k}\right)=\left|Y_{2,(i j)}\left(s_{k}\right)\right|^{2}
\end{array}
$$

where $K_{s}, P_{\text {in }}$ and $P_{\text {out }}$ are the number of frequency samples, input and output ports of the system, respectively.

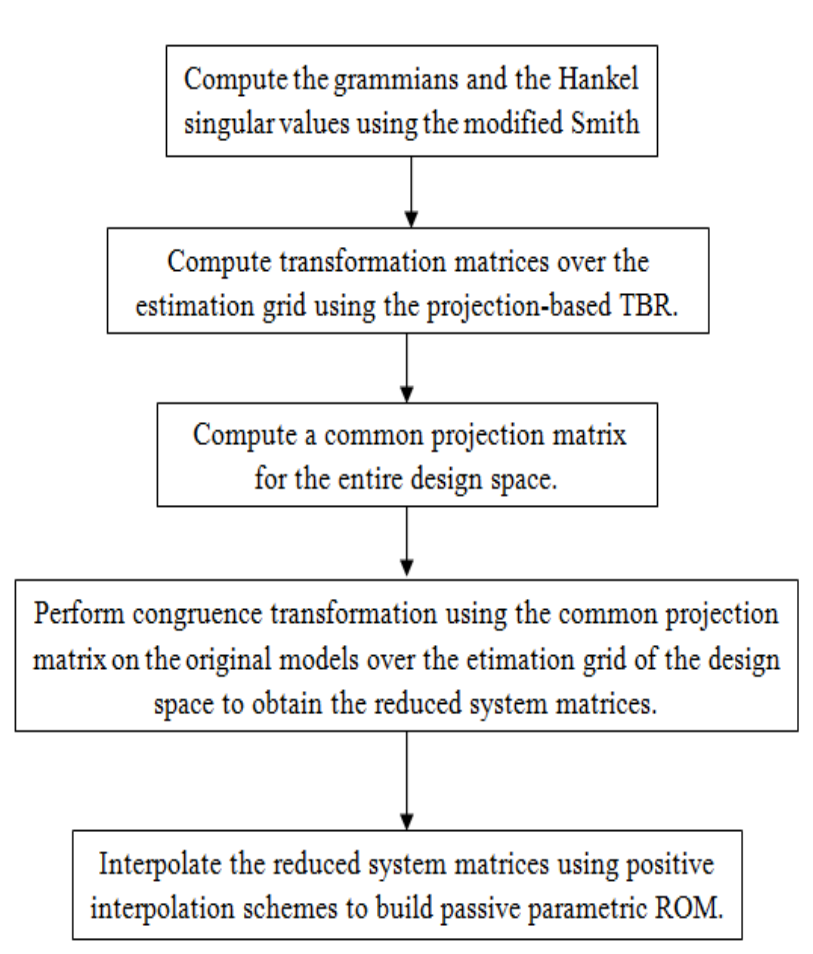

Fig. 2. Flowchart of the proposed technique.

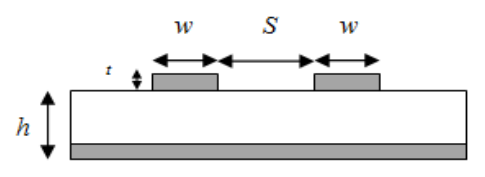

Fig. 3. EX1: Cross section of two coupled microstrip lines.

5.1 EX1: Two coupled microstrips with one parameter variation in addition to frequency

Table 1. PARAMETERS OF TWO COUPLED MICROSTRIPS

\begin{tabular}{|l|l|l|}
\hline Parameter & Min & Max \\
\hline Frequency (freq) & $1 \mathrm{kHz}$ & $4 \mathrm{GHz}$ \\
Length $(L)$ & $2 \mathrm{~cm}$ & $6 \mathrm{~cm}$ \\
\hline
\end{tabular}

Two coupled microstrips are modeled as described in, Knockaert and De Zutter [2000]. Fig.3 shows their cross section. The conductors have width $w=100 \mu \mathrm{m}$ and thickness $t=50 \mu \mathrm{m}$. The length $L$ is considered as a parameter in addition to frequency. Their corresponding ranges are shown in Table 1 . The $\mathbf{C}, \mathbf{G}, \mathbf{B}, \mathbf{L}$ matrices are obtained for 5 values of $L$. The original models are represented as in (1) satisfying the conditions in (18) and have an order of 2002. The matrices $\mathbf{C}$ and $\mathbf{G}$ can be represented as:

$$
\mathbf{C}=\left[\begin{array}{ll}
\mathbf{Q} & 0 \\
0 & \mathbf{H}
\end{array}\right] \quad \mathbf{G}=\left[\begin{array}{cc}
\mathbf{N} & \mathbf{E} \\
-\mathbf{E} & \mathbf{R}
\end{array}\right]
$$

where $\mathbf{Q}, \mathbf{H}, \mathbf{R}$ and $\mathbf{N}$ are the capacitance, inductance, resistance and conductance matrices respectively and $\mathbf{E}$ is the incident matrix associated with the connectivity.

$L=\{2,4,6\} \mathrm{cm}$ are considered as the estimation points and $L=\{3,5\} \mathrm{cm}$ are considered as the validation points. The 


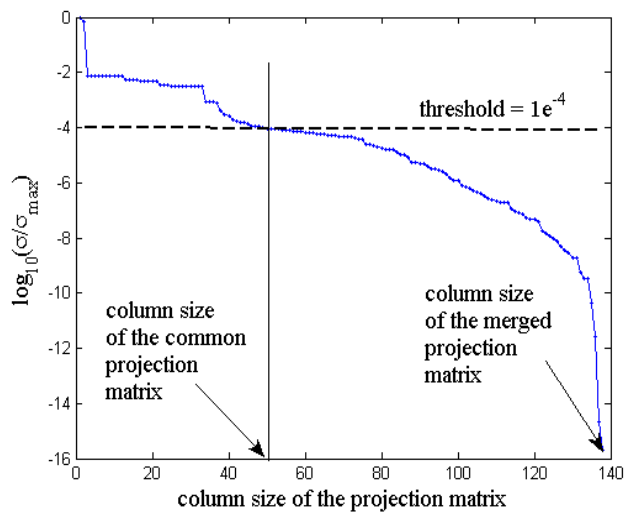

Fig. 4. EX1: Singular values of the projection matrix.

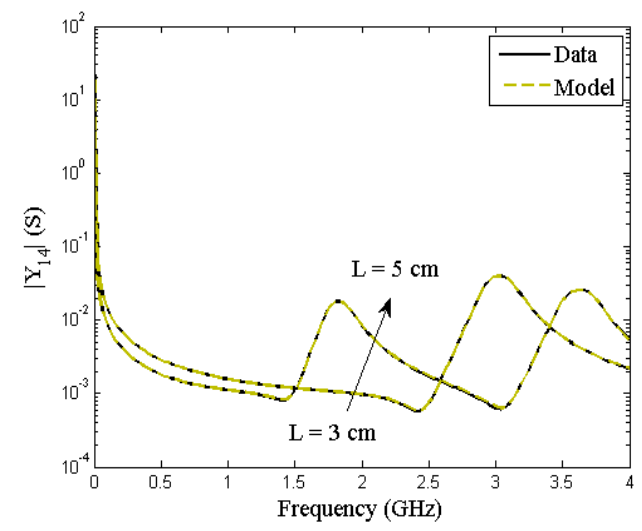

Fig. 5. EX1: Magnitude of $\mathbf{Y}_{14}(s, L)$ for $L=\{3,5\} \mathrm{cm}$ using a common projection matrix.

reduced order and the transformation matrices are computed for the estimation points as described in Section 2. Then a common projection matrix is computed for the entire design space. If the union of the transformation matrices had been used for the congruence transformation, then the reduced order would have been 138 . Due to the truncation of the singular values by a threshold of $1 e-4$ the dimension of the common projection matrix is 50 as shown in Fig. 4. Fig.5 compares $\mathbf{Y}_{14}(s, L)$ and its reduced order for the validation points for length $L=$ $\{3,5\} \mathrm{cm}$. The weighted RMS error is equal to 0.014 . These specific points have not been used for the reduced order model generation. The parametric ROM is able to accurately describe the parametric behavior of the system with a small order. Overall passivity is guaranteed by construction.

\subsection{EX2: Two coupled microstrips with two parameter variation in addition to frequency}

Microstrips are studied in this example considering two parameters in addition to frequency, namely the length of the lines $L$ and a fabrication parameter $\lambda$. The matrices $\mathbf{C}$ and $\mathbf{G}$ can be written as

$$
\mathbf{C}(\lambda)=\left[\begin{array}{cc}
(1+\lambda) \mathbf{Q} & 0 \\
0 & \mathbf{H}
\end{array}\right] \quad \mathbf{G}(\lambda)=\left[\begin{array}{cc}
\mathbf{N} & \mathbf{E} \\
-\mathbf{E} & (1+\lambda) \mathbf{R}
\end{array}\right]
$$

where $\lambda$ represents the variations in the fabrication parameter and affects the capacitance and resistance matrices, $\mathrm{Li}$ et al. [2008]. Their corresponding ranges are shown in Table
Table 2. PARAMETERS OF TWO COUPLED MICROSTRIPS

\begin{tabular}{|l|l|l|}
\hline Parameter & Min & Max \\
\hline Frequency $($ freq) & $1 \mathrm{kHz}$ & $4 \mathrm{GHz}$ \\
Length $(L)$ & $2 \mathrm{~cm}$ & $6 \mathrm{~cm}$ \\
Fabrication Parameter $(\lambda)$ & $-15 \%$ & $15 \%$ \\
\hline
\end{tabular}

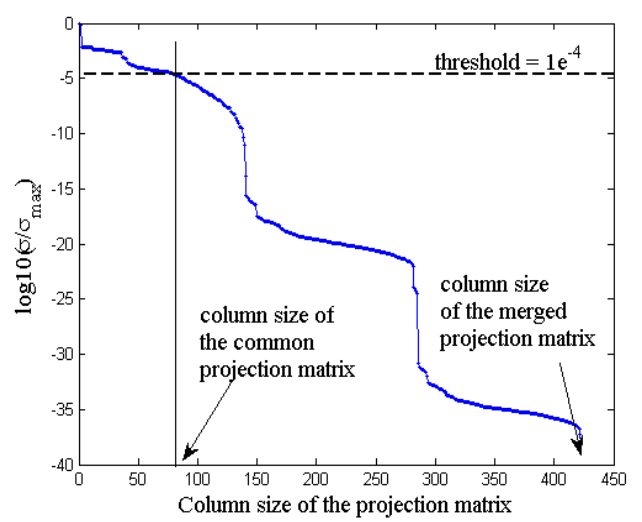

Fig. 6. EX2: Singular values of the projection matrix.

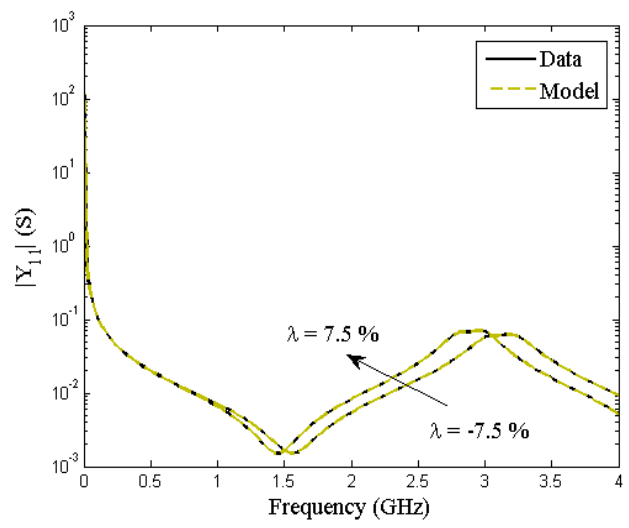

Fig. 7. EX2: Magnitude of $\mathbf{Y}_{11}(s, L, \lambda)$ for $\lambda=\{-7.5,7.5\} \%$ $L=3 \mathrm{~cm}$ using a common projection matrix.

2. A $3 \times 3(L, \lambda)$ estimation grid, $L=\{2,4,6\} \mathrm{cm}$ and $\lambda=$ $\{-15,0,15\} \%$ is considered and a validation grid of $2 \times 2$ $(L, \lambda), L=\{3,5\} \mathrm{cm}$ and $\lambda=\{-7.5,7.5\} \%$ are considered. The reduced order and the transformation matrices are computed at the estimation points as described in Section 2.

A common projection matrix is obtained for the entire design space. Similarly, to the previous example,the truncation of the singular values leads to obtain a small reduced order, as shown in Fig.6. Fig.7 compares $\mathbf{Y}_{11}(s, L, \lambda)$ and its reduced order for the validation points for $\lambda=\{-7.5,7.5\} \%$ and $L=3 \mathrm{~cm}$. The weighted RMS error is equal to 0.017 . This specific point have not been used for the reduced order model generation.

It can be noted that even with more than one parameter in addition to frequency, the parametric ROM is able to accurately captures the dynamic system behavior that is influenced by the design parameters. As in the previous example, the passivity of the parametric ROM is guaranteed over the entire design space. 


\section{CONCLUSION}

We have presented a robust PMOR method using matrix interpolation and a common projection matrix. The design space is sampled over an estimation grid and transformation matrices are computed at the estimation points using a projection-based passive TBR. A common projection matrix is obtained by the truncation of the singular values of the merged transformation matrices obtained at the estimation points in the design space. The reduced system matrices are then interpolated using positive interpolation schemes to obtain a passive parametric reduced model. Pertinent numerical examples show that the presented PMOR is able to build accurate parametric reduced models that guarantee passivity.

\section{REFERENCES}

E. A. Guillemin. Synthesis of Passive Networks. Wiley, New York, 1957.

E. W. Cheney. Multivariate approximation theory : Selected topics. CBMS NSF Regional Conference Series in Applied Mathematics, 51, Philadelphia, PA : SIAM, Dec. 1986.

L. Daniel, Ong Chin Siong, L.S. Chay, Kwok Hong Lee, and J. White. A multiparameter moment-matching modelreduction approach for generating geometrically parameterized interconnect performance models. IEEE Transactions on Computer-Aided Design of Integrated Circuits and Systems, 23(5):678 - 693, May 2004.

R. Eid, R. Castane-Selga, H. Panzer, T. Wolf, and B. Lohmann. Stability-preserving parametric model reduction by matrix interpolation. Mathematical and Computer Modeling of Dynamical Systems, 17(4):319-335, 2011.

O. Farle, S. Burgard, and R. Dyczij-Edlinger. Passivity preserving parametric model-order reduction for non-affine parameters. Mathematical and Computer Modelling of Dynamical Systems, 17(3):279-294, 2011.

P. Feldmann and W. R. Freund. Efficient linear circuit analysis by Padè approximation via the Lanczos process. IEEE Transactions on Computer-Aided Design of Integrated Circuits and Systems, 14(5):639-649, May 1995.

F. Ferranti, G. Antonini, T. Dhaene, and L. Knockaert. Guaranteed passive parameterized model order reduction of the partial element equivalent circuit (PEEC) method. IEEE Transactions on Electromagnetic Compatibility, 52(4):974984, Nov. 2010.

F. Ferranti, G. Antonini, T. Dhaene, L. Knockaert, and A.E. Ruehli. Physics-based passivity-preserving parameterized model order reduction for PEEC circuit analysis. IEEE Transactions on Components, Packaging and Manufacturing Technology, 1(3):399-409, Mar. 2011.

Roland W. Freund. Krylov-subspace methods for reduced-order modeling in circuit simulation. J. Comput. Appl. Math., 123: 395-421, Nov. 2000. ISSN 0377-0427.

K. Gallivan, E. Grimme, and Van P. Dooren. Asymptotic waveform evaluation via a Lanczos method. Applied Mathematics Letter, 7(5):75-80, Sept. 1994.

K. Gallivan, E. Grimme, and P. Van Dooren. A rational Lanczos algorithm for model reduction. Numerical Algorithms, 12(1): 33-63, Mar. 1996.

Keith Glover. All optimal hankel-norm approximations of linear multivariable systems and their $\mathrm{L}^{\infty}$-error bounds. International Journal of Control, 39(6):1115-1193, 1984.
S. Gugercin, C. D. Sorensen, and C. A. Antoulas. A modified low-rank smith method for large scale lyapunov equations. Numerical Algorithms, 32(1):27-55, May 2001.

P. K. Gunupudi, R. Khazaka, M.S. Nakhla, T. Smy, and D. Celo. Passive parameterized time-domain macromodels for highspeed transmission-line networks. IEEE Transactions on Microwave Theory and Techniques, 51(12):2347 - 2354, Dec. 2003.

M. Kamon, F. Wang, and J. White. Generating nearly optimally compact models from krylov-subspace based reduced-order models. IEEE Transactions on Circuits and Systems II: Analog and Digital Signal Processing, 47(4):239 -248, Apr. 2000.

L. Knockaert and D. De Zutter. Laguerre-SVD reduced-order modeling. IEEE Transactions on Microwave Theory and Techniques, 48(9):1469-1475, Sept. 2000.

A.T.-M. Leung and R. Khazaka. Parametric model order reduction technique for design optimization. pages $1290-$ 1293 Vol. 2, may 2005.

Peng Li, Frank Liu, Xin Li, Lawrence T. Pileggi, and Sani R. Nassif. Modeling interconnect variability using efficient parametric model order reduction. 2:958-963, 2005.

Yung-Ta Li, Zhaojun Bai, Yangfeng Su, and Xuan Zeng. Model order reduction of parameterized interconnect networks via a two-directional arnoldi process. IEEE Transactions on Computer-Aided Design of Integrated Circuits and Systems, 27(9):1571 -1582, Sept. 2008.

A. Odabasioglu, M. Celik, and L.T. Pileggi. PRIMA: passive reduced-order interconnect macromodeling algorithm. IEEE Transactions on Computer-Aided Design of Integrated Circuits and Systems, 17(8):645 -654, Aug. 1998.

H. Panzer, J. Mohring, R. Eid, and B. Lohmann. Parametric model order reduction by matrix interpolation. Automatisierungstechnik, pages 475-484, Aug. 2010.

R. Rohrer and H. Nosrati. Passivity considerations in stability studies of numerical integration algorithms. International Journal of Control, 28(9):857 - 866, Sept. 1981.

D. F. Watson. Computing the n-dimensional delaunay tessellation with application to voronoi polytopes. The Computer Journal, 24(2):167-172, Feb. 1981.

L. Weinberg. Network Analysis and Synthesis. McGraw-Hill Book Company, New York, 1962.

Alan Weiser and Sergio E. Zarantonello. A note on piecewise linear and multilinear table interpolation in many dimensions. Mathematics of Computation, 50(181):189 - 196, Jan. 1988.

Ngai Wong, V. Balakrishnan, Cheng Kok Koh, and Tung Sang Ng. Two algorithms for fast and accurate passivitypreserving model order reduction. IEEE Transactions on Computer-Aided Design of Integrated Circuits and Systems, 25(10):2062 -2075, Oct. 2006.

Boyuan Yan, Sheldon X.-D. Tan, Pu Liu, and Bruce McGaughy. Passive interconnect macromodeling via balanced truncation of linear systems in descriptor form. Asia and South Pacific Design Automation Conference, pages 355-360, 2007. 